\title{
Hybrid Oleate-lodide Ligand Shell for Air-Stable PbSe Nanocrystals and Superstructures
}

\author{
J. L. Peters, ${ }^{\dagger}$ J. C. van der Bok, ${ }^{\dagger}$ J. P. Hofmann, ${ }^{\ddagger}$ and D. Vanmaekelbergh ${ }^{*}{ }^{\dagger}$ \\ ${ }^{\dagger}$ Condensed Matter and Interfaces, Debye Institute for Nanomaterials Science, Utrecht University, P.O. Box 80000, 3508 TA \\ Utrecht, The Netherlands \\ ${ }^{\dagger}$ Laboratory for Inorganic Materials and Catalysis, Department of Chemical Engineering and Chemistry, Eindhoven University of \\ Technology, P.O. Box 513, 5600 MB Eindhoven, The Netherlands
}

\section{Supporting Information}

ABSTRACT: A postsynthetic treatment is presented to improve the air stability of PbSe nanocrystals (NCs) and PbSe square superstructures. The addition of z-type $\mathrm{Pb}$ (oleate) $)_{2}$ ligands together with $\mathrm{x}$-type iodide ligands creates a hybrid ligand shell containing both ligands. The air stability of the $\mathrm{PbSe} \mathrm{NCs}$ is checked by enduring absorption spectroscopy under ambient conditions. With a combined $\mathrm{NaI}+\mathrm{Pb}$ (oleate) $)_{2}$ treatment, the absorption spectrum remains unchanged for several days under ambient conditions. Fourier transform infrared spectroscopy shows that the surface coordination of the oleate ligands changes by the chemical treatment: from mixed chelating
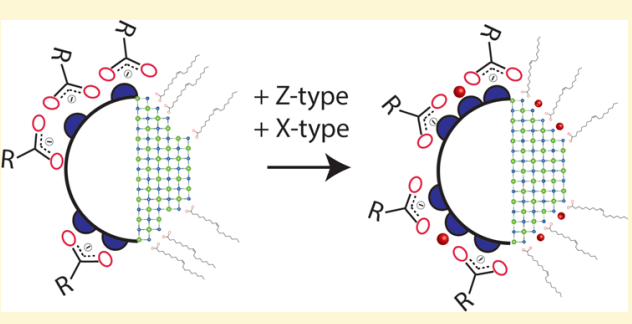
bidentate + bridging to $\mathrm{Pb}$ for the pristine nanocrystals to almost exclusive chelating bidentate coordination after chemical passivation. The shift of the $\mathrm{C}-\mathrm{H}$ stretching vibration shows that the oleate hydrocarbon layer is in a more liquidlike state after the chemical treatment, suggesting that oleate and iodide ligands are often present on adjacent surface positions.

\section{INTRODUCTION}

Lead chalcogenide colloidal nanocrystals (NCs) are of great interest as they possess a small band gap and a large exciton Bohr radius. ${ }^{1}$ As a result, they show bright and strongly sizetunable luminescence over the complete near-infrared region in frequency regions that are important for data processing and biological labeling. Moreover, PbSe NCs show a high carrier multiplication efficiency, of interest for several optoelectronic devices. $^{2-6}$ Lead chalcogenide colloidal nanocrystals also showed the propensity to self-organize in two dimensions followed by oriented attachment, forming several superstructures wherein the NCs are electronically coupled. ${ }^{7-9}$ The coupling results in a specific electronic band structure, determined by the degree of coupling and the nanoscale geometry of the superstructure. ${ }^{10,11}$

Besides these promises for optoelectronics, the chemistry of the lead chalcogenides is complex and not application-friendly, as they are very vulnerable for chemical oxidation in ambient atmosphere, resulting in irreversible changes of the optoelectronic properties. ${ }^{12}$ Several approaches for air-stable $\mathrm{Pb}$ chalcogenide nanocrystals have been published, for example, the growth of a cadmium-containing inorganic shell ${ }^{13,14}$ or treatment with $\mathrm{Cl}_{2}{ }^{15}$ Although these methods increase the chemical stability, they also alter the NC facets, making the $\mathrm{PbSe}$ NCs unsuitable as building blocks for superstructures. Another approach is the addition of halide-containing precursors $^{5,16,17}$ or halide salts ${ }^{6,18}$ during the aftermath of the NC synthesis. This resulted in air-stable NCs, but these methods cannot be used for two-dimensional (2D) PbSe superstructures.
Here, we introduce a method for chemical passivation applicable for dispersed $\mathrm{PbSe} \mathrm{NCs}$ and $\mathrm{PbSe}$ superstructures as well. The method consists of the addition of $x$-type iodide ligands, which exchange the oleate ligands, combined with the intentional addition of z-type $\mathrm{Pb}$ (oleate) $)_{2}$ ligands. We show that the PbSe NCs obtain a hybrid ligand shell composed of both iodide and oleate ligands bound to surface $\mathrm{Pb}$ ions. The air stability is confirmed by absorption spectroscopy and X-ray photoelectron spectroscopy (XPS). While regular nanocrystal assembly and oriented attachment are carried out under oxygen-free conditions in a glovebox, the air-stable nanocrystals allowed us to perform assembly and attachment experiments in ambient atmosphere, yielding linear superstructures. Furthermore, the combined treatment can also be performed on the superstructures themselves. In this way, the effects of oxidation on square superstructures are delayed remarkably after the chemical surface treatment. Fourier transform infrared (FT-IR) spectroscopy was used to show that the carboxylate ligands on air-stable PbSe NCs are almost exclusively bound in a chelating bidentate coordination to lead. We introduce and use a new $\mathrm{C}-\mathrm{H}$ stretch peak analysis that shows that in the hybrid oleate-iodide ligand shell the oleate ligands are in a more liquid state (have more freedom of motion) than in a pure oleate ligand shell. This hints to a molecular mixing of the iodide and oleate moieties in the hybrid shells.

Received: May 14, 2019

Revised: June 27, 2019

Published: July 9, 2019 


\section{RESULTS}

Stability Analysis by Optical Absorption Spectroscopy. We adapted the method from Woo et al. ${ }^{18}$ to make airstable NCs after the NC synthesis and purification: we dropwise added a $\mathrm{NaI}$ solution in methanol to a purified $\mathrm{PbSe}$ $\mathrm{NC}$ dispersion and intentionally add extra $\mathrm{Pb}$ (oleate) $)_{2}$ ligands (see the Experimental Section for details). The experiments on the surface passivation against oxidation have been performed with $\mathrm{PbSe}$ nanocrystals from different batches. We also have used two different synthesis methods (see methods). We observed that the passivation against oxidation does not depend on the batch of nanocrystals that we used nor on the synthesis method (see Figure S1). We evaluated possible oxidation of untreated and chemically treated $\mathrm{PbSe}$ nanocrystals in ambient by forming a thin, vacuum-dried, nanocrystal film, which is exposed for 3 days to ambient atmosphere. The electronic structure of the nanocrystals is measured by absorption spectroscopy as shown in Figure 1. The absorption

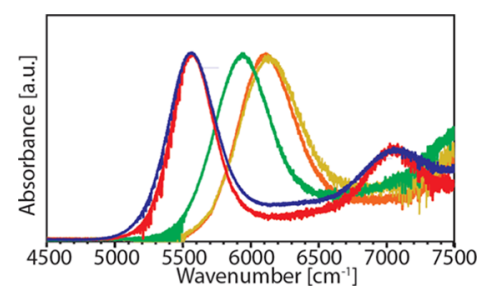

Figure 1. Stability in ambient for differently treated $\mathrm{PbSe}$ nanocrystals assessed by optical absorption spectroscopy. The figure shows absorption spectra of PbSe NCs, $6.3 \mathrm{~nm}$ in size, all originating from the same synthesis. Blue: spectrum of a dispersion of untreated $\mathrm{PbSe}$ NCs, measured under oxygen-free conditions. Other colors: $\mathrm{PbSe}$ NCs washed with methanol, centrifuged, dried, and subsequently put in ambient atmosphere for 3 days. Yellow: untreated NCs strongly blue-shifted due to oxidation after keeping for 3 days in ambient; orange: $\mathrm{PbSe} \mathrm{NCs}$ washed with solely $\mathrm{Pb}$ (oleate) ${ }_{2}$ added to the $\mathrm{NC}$ suspension, strongly oxidized after keeping for 3 days in ambient; green: $\mathrm{PbSe} \mathrm{NCs}$ washed with $\mathrm{NaI}$ (dissolved in methanol), and red: $\mathrm{PbSe}$ NCs washed with $\mathrm{NaI}$ in methanol and additional $\mathrm{Pb}$ (oleate $)_{2}$.

spectrum of untreated $\mathrm{PbSe}$ nanocrystals, kept under oxygenfree conditions, is taken as a reference. A film of untreated NCs shows a strong blue shift of the first absorption peak, indicative of a diminishing $\mathrm{PbSe}$ nanocrystal volume by surface oxidation (chemical details follow below). ${ }^{12}$ The red curve in Figure 1 shows the exciton peak of this NC film on the exactly same position as for pristine $\mathrm{PbSe} \mathrm{NCs}$, demonstrating full stability of the NCs treated in this way. Transmission electron microscopy (TEM) images of both the original and chemically treated NCs exposed to ambient are shown in Figure S2.

To be able to measure the action of the iodide and $\mathrm{Pb}$ (oleate $)_{2}$ components separately, we added either only $\mathrm{Pb}$ (oleate) $)_{2}$ (Figure 1, orange curve) or only $\mathrm{NaI}$ (Figure 1, green curve) to the purified $\mathrm{PbSe} \mathrm{NC}$ suspension in toluene. Treatment of the dispersion with only NaI did not prevent NC oxidation, as demonstrated by a considerable blue shift of the absorption peak. Even worse, addition of only $\mathrm{Pb}(\text { oleate })_{2}$ did not show any improvement in the stability of the PbSe NCs. This shows that it is the combination of both oleate and iodide ligands that make the NCs air-stable. We note that the full width at half-maximum becomes slightly smaller (from 57 to $45 \mathrm{meV}$ ) after the chemical treatment with respect to pristine nanocrystals. A similar result has been observed before. ${ }^{19}$
For a given NC size and concentration, there is an optimum iodide concentration that yields completely air-stable NCs. This optimum was found to be approximately 0.8 times the amount of oleate ligands already attached to pristine NC surfaces. This amount is determined by FT-IR and nuclear magnetic resonance (NMR) spectroscopies, using procedures published before. ${ }^{20-22}$ Too much iodide results in aggregation of the NCs and too little iodide in PbSe NCs that are only partially air-stable, as visible from an absorption peak shift after exposure to ambient conditions. $\mathrm{Pb}$ (oleate) $)_{2}$ should be added in at least the same concentration as iodide, but higher concentrations did not have an influence on the results.

Chemical Surface Analysis by X-ray Spectroscopy (EDX) and XPS. To verify the presence of iodide on the NC surface, we used energy-dispersive X-ray spectroscopy (EDX) and X-ray photoelectron spectroscopy (XPS) on the air-stable NCs; see Figure 2 as well as Figures S3 and S4. While iodide

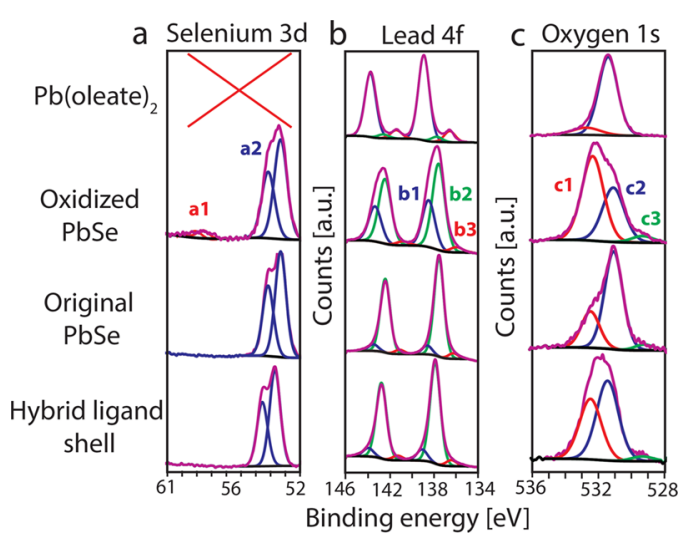

Figure 2. Stability in ambient for differently treated PbSe nanocrystals monitored from the surface composition measured by XPS. On the vertical axis, we indicate the different samples: first, $\mathrm{Pb}$ (oleate) $_{2}$; second, oxidized $\mathrm{PbSe}$; third, pristine $\mathrm{PbSe} \mathrm{NCs}$; and fourth, $\mathrm{PbSe}$ NCs with hybrid oleate/iodide shell. The three different columns show the XPS core-level spectra for (a) Se 3d, (b) Pb 4f, and (c) O 1s. The measured signal is shown in purple, and the background is in black. The fitted components are in blue, red, and green and are discussed in the main text.

peaks can be clearly observed in both EDX and XPS spectra (Figures S3 and S4, respectively), sodium could not be detected. The absence of sodium on the NC surface shows that iodide acts as an $\mathrm{x}$-type ligand and exchanges with oleate on the NC surface. The $x$-type ligand exchange preserves the required charge neutrality of each nanocrystal dispersed in an apolar solvent. It is also not surprising that the exchange of too much oleate for iodide results in insoluble NCs, since hydrocarbon chains are necessary for the solubility of the NCs in the apolar toluene solvent.

The role of $\mathrm{Pb}$ (oleate) $)_{2}$ can be twofold. It can either act as a z-type ligand itself attaching to a surface Se, or it can react with iodide to form $\mathrm{PbI}_{2}$, which subsequently could also act as a $\mathrm{z}$ type ligand on the NC surface. Either way, it is required to add a source of neutral z-type ligands to obtain complete chemical stability. This is in line with EDX results, suggesting an increase of the $\mathrm{Pb} / \mathrm{Se}$ ratio for $6.1 \mathrm{~nm} \mathrm{NCs}$, from 1.1 for untreated to 1.2 for treated NCs. These findings suggest that a complete surface coverage of the $\mathrm{Pb}$ atoms on a given facet can occur by a mixture of smaller iodide ligands, giving enough room for the more extended oleate chains. 

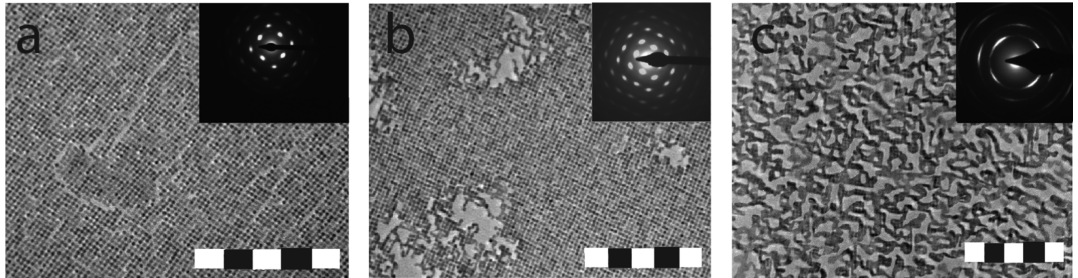

Figure 3. Effect of oxygen on the quality of $2 \mathrm{D}$ PbSe NC superstructures. TEM images of square superstructures, self-assembled and attached in a glovebox in which different $\mathrm{O}_{2}$ concentrations were present. (a) $\mathrm{O}_{2}$ concentration lower than $3 \mathrm{ppm}$, (b) $\mathrm{O}_{2}$ concentration approximately 8 ppm, and (c) $\mathrm{O}_{2}$ concentration $>15 \mathrm{ppm}$. Electron diffraction patterns in the insets show that all of the NCs are oriented in the same way and have their (100) facets upward. Scale bar is $200 \mathrm{~nm}$.
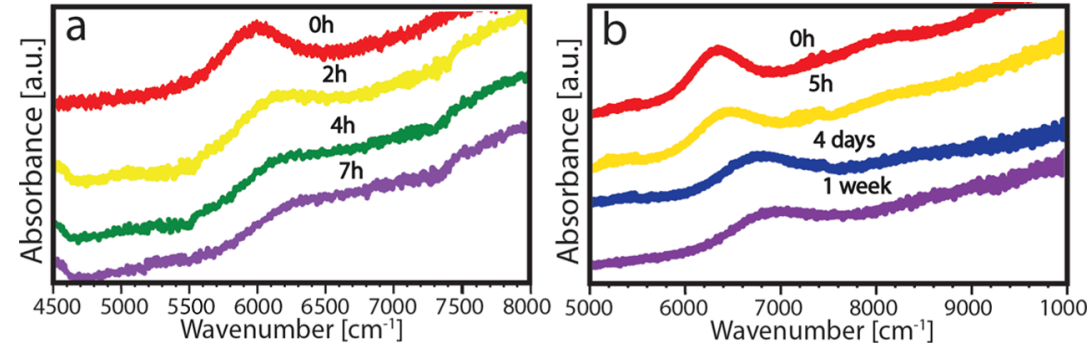

Figure 4. Diminution of the exciton peak on a square superstructure after exposure to ambient atmosphere. Oxidation on (a) a superstructure made from pristine NCs and (b) for superstructures protected after the formation. The time intervals are indicated in the picture. The superstructure made from pristine NCs immediately shows a shift and a decrease in intensity, while for the protected superstructure, this is delayed substantially.

To study the air stability in more detail, we measured XPS on $\mathrm{Pb}$ (oleate $)_{2}$, oxidized NCs, and air-stable NCs exposed to ambient conditions (Figure 2) and compared the results with those of pristine, unexposed $\mathrm{PbSe}$ nanocrystals. The Se $3 \mathrm{~d}$ core level is spin-orbit split into two peaks, $3 \mathrm{~d}_{3 / 2}$ and $3 \mathrm{~d}_{5 / 2}$, with a splitting of $0.86 \mathrm{eV}$. The peak $(\mathrm{a} 2)$ at $\mathrm{BE}\left(\mathrm{Se} 3 \mathrm{~d}_{5 / 2}\right)=53.3 \mathrm{eV}$ represents $\mathrm{Se}^{2-}$ in the core of the PbSe NCs. ${ }^{12,23,24}$ Another doublet appears for the oxidized NCs at a Se $3 \mathrm{~d}_{5 / 2}$ binding energy of $58.3 \mathrm{eV}$ (a1), corresponding to oxidized Se surface species. This oxidation state points to the presence of $\mathrm{SeO}_{2}$ or $\mathrm{SeO}_{3}{ }^{2-}$ moieties at the NC surface. ${ }^{12,25-27}$ In the case of airstable NCs as well as the unexposed PbSe NCs, these species could not be detected. This, together with the results from absorption spectroscopy, shows the unchanged surface composition and air stability of the NCs treated after applying a mixture of iodide and $\mathrm{Pb}$ (oleate) $)_{2}$.

The $\mathrm{Pb} 4 \mathrm{f}_{7 / 2}$ and $4 \mathrm{f}_{5 / 2}$ core-level spectra (Figure $2 \mathrm{~b}$ ) were fitted by at least three components. The blue, higher-bindingenergy peak $\left(\mathrm{b} 1, \mathrm{BE}\left(\mathrm{Pb} 4 \mathrm{f}_{7 / 2}\right)=138.5 \mathrm{eV}\right)$ is attributed to oxidation products and is presumably combined with lead bound to oleate, iodide, and possibly $\mathrm{PbSeO}_{3}$ species. ${ }^{12}$ The peak (b2) at $\mathrm{BE}\left(\mathrm{Pb} 4 \mathrm{f}_{7 / 2}\right)=137.6 \mathrm{eV}$ is related to the $\mathrm{Pb}^{2+}$ in the PbSe core. ${ }^{28}$ The peak (b3) at $\mathrm{BE}\left(\mathrm{Pb} 4 \mathrm{f}_{7 / 2}\right)=136.4 \mathrm{eV}$ shows that there is a small quantity of metallic lead on the NC surface $^{29}$ as well as in the oleate precursor. The origin of this is not yet clear.

The interpretation and assignment of the $\mathrm{O}$ 1s core-level region are complex because of strongly overlapping peaks and similar binding energies for a variety of different species. $\mathrm{Pb}$ (oleate) $)_{2}$ has two main species, a carboxylate at $531.5 \mathrm{eV}$ and an acid impurity at $532.7 \mathrm{eV}$, which are both confirmed by FT-IR spectroscopy (see Figure S5). The oxygen peaks of the $\mathrm{NCs}$ are less clear, but the peak (c3) at $\mathrm{BE}(\mathrm{O} 1 \mathrm{~s})=529.2 \mathrm{eV}$ seems to originate from lead oxide. ${ }^{30,31}$ The peak (c2) at around $531.0 \mathrm{eV}$ is often attributed to hydroxide, ${ }^{32,33}$ but this is rather disputable since the peak is at a similar position as the carboxylate, in the reference $\mathrm{Pb}$ (oleate) $)_{2}$ sample. We should remark that there is also no sign for the presence of hydroxide in our FT-IR spectra (see Figure S6). ${ }^{34}$ Peaks (c1) and (c2) most likely originate from bound carboxylate (carboxylic acid is not present in the PbSe NCs).

Chemical Surface Passivation of 2D PbSe NC Superstructures. It became clear that the formation of $2 \mathrm{D}$ atomically coherent square and honeycomb superstructures by assembly and attachment of PbSe NCs is a delicate process very vulnerable to traces of oxygen in the reactor, in our case, simply the glovebox. An example of the destructive influence of oxygen during the formation of square superlattices is shown in Figure 3. Electron diffraction shows atomic alignment at all three oxygen concentrations, but the square nanoscale geometry is severely damaged at oxygen levels of $8 \mathrm{ppm}$ and completely destroyed above $15 \mathrm{ppm}$. Despite the disappearance of the long-range nanoscale order, there is still some local atomic coherency. This suggests that oriented attachment between the PbSe NCs via the (100) facets still occurs to some extent, even when small amounts of oxygen are present; yet, the destructive role of oxygen for the long-range order is not fully understood. ${ }^{35}$ There are two ways to make superstructures air-stable: first, use of PbSe NCs that are chemically treated for air stability in the formation of superstructures and second, use of pristine $\mathrm{PbSe} \mathrm{NCs}$ and application of the chemical treatment on the superstructure after formation.

With the chemically treated air-stable NCs, self-assembly can be performed in ambient conditions, in principle. The procedure of self-assembly and oriented attachment in ambient resulted in linear structures in which nanocrystals were (100)/ (100) facet-connected (Figure S7). From the structures that we observe, we can conclude that the presence of oxygen does not have the destructive effects visible as for untreated NCs in Figure 3. We also performed the process of assembly and oriented attachment with air-stable NCs in a glovebox, which resulted in small patches of square superstructures (Figure S8). 
The amount of $(100) /(100)$ epitaxial connections is smaller than that of untreated PbSe nanocrystals, and also the superlattice domain size is smaller. It is not known if an optimization for more perfect square superlattices is feasible with these surface-passivated nanocrystals.

$\mathrm{PbI}_{2}$ is hardly soluble in ethylene glycol, which makes it unlikely that it detaches from the NC surface. Besides that, lead iodide has a hexagonal crystal structure, which fits better on the hexagonal packing of lead atoms on the (111) facets compared to a square packing on the (100) facets. ${ }^{36}$ This makes us believe that the (100) facets, which are exclusively used for oriented attachment, are not fully covered with $\mathrm{PbI}_{2}$ as a z-type ligand. The same NCs are air-stable, which suggests that (100) protection is not necessary for their air stability.

As a second method, we formed nanocrystal superstructures from pristine $\mathrm{PbSe} \mathrm{NCs}$ by the common procedure in an oxygen-free glovebox and applied the chemical treatment $(\mathrm{NaI}$ and $\left.\mathrm{Pb}(\text { oleate })_{2}\right)$ for air stability on the formed superstructures. A TEM image of the superstructure after the treatment is shown in Figure S9, and the air stability experiments are presented in Figure 4. It is clear that the chemical passivation after the superstructure formation is most successful: the amplitude of the absorption related to the exciton resonance diminishes much slower compared to that for untreated superstructures. This confirms that the chemical passivation with $\mathrm{NaI}$ and $\mathrm{Pb}$ (oleate) ${ }_{2}$ can be applied to both individual nanocrystals and extended solid-state systems. Dried or dispersed nanocrystals generally have longer air stability (see Figure 1), which we attribute to the much less oxygen-tosurface exposure compare to single NC thick superstructures.

Surface Passivation by Other Chemical Treatments. We have seen that sodium does not end up on the NC surface. We therefore tried to protect $\mathrm{PbSe} \mathrm{NCs}$ with other salts as well, for example, $\mathrm{NaCl}, \mathrm{NH}_{3} \mathrm{X}, \mathrm{PbCl}_{2}, \mathrm{PbBr}_{2}$, and $\mathrm{CdX}_{2}(X=$ $\mathrm{Cl}, \mathrm{Br}, \mathrm{I})$. The results are summarized in Table S1. Protecting $\mathrm{PbSe}$ NCs with $\mathrm{CdX}_{2}$, however, impedes oriented attachment in the self-assembly of superstructures (Figure S10).

Oleate Coordination. A detailed picture of the surface chemistry of pristine PbSe NCs and chemically passivated NCs is obtained from FT-IR spectroscopy. Figure 5a shows the carboxylate stretch region, where the spectral intensity has been normalized for the number of oleate ligands. The spectral shift $(\Delta v)$ between the symmetric ( $v 1)$ and the asymmetric (v2) carboxylic stretch indicates the bonding coordination of the carboxylic group to the surface $\mathrm{Pb}$ atoms. ${ }^{20,37-41}$ Peaks v1

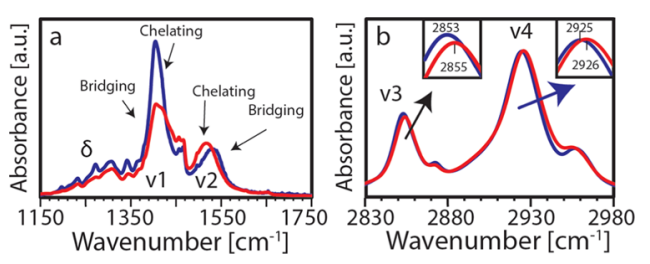

Figure 5. Structure of the oleate ligand shell for PbSe NCs with a hybrid iodide/oleate ligand shell (red) or original PbSe NCs with only oleate ligands (blue) measured by FT-IR spectroscopy. (a) FTIR spectra showing the $\mathrm{CH}_{2}$ bending $(\delta)$, symmetric (v1), and asymmetric (v2) carboxylic stretching vibrations. The energy difference $(\Delta \mathrm{v})$ between the symmetric (v1) and asymmetric (v2) carboxylic stretches indicates the carboxylic coordination to lead. (b) Symmetric (v3) and asymmetric $\mathrm{C}-\mathrm{H}$ stretch vibrations (v4). The red curve is slightly blue-shifted, indicating more fluidlike hydrocarbon chains for a hybrid ligand shell. and v2 are doublets, which are split by fitting two Gaussians, which shows that there are two different oleate coordinations. The deconvolution and characteristics of each peak are shown in Figure S11 and Table S2. The energy difference representing the two carboxylic coordinations is shown in Table 1 . The combination of the lower-energy symmetric (v1)

Table 1. Overview of the Peak Differences of the Carboxylate Vibrations for the Untreated and Air-Stable NCs

\begin{tabular}{lcc} 
& $\Delta v_{\text {chelating }}\left(\mathrm{cm}^{-1}\right)$ & $\Delta v_{\text {bridging }}\left(\mathrm{cm}^{-1}\right)$ \\
untreated $\mathrm{PbSe}$ & 104 & 133 \\
hybrid ligand shell & 92 & 124 \\
\hline
\end{tabular}

peak with the higher-energy asymmetric (v2) peak is an indication of a bridging bidentate carboxylate coordination, while that of the higher-energy symmetric (v1) peak with the lower-energy asymmetric (v2) peak $\left(\Delta \mathrm{v}_{\text {outer }}\right)$ represents a chelating bidentate carboxylic coordination. ${ }^{20,37}$

The decrease in the intensity of the symmetric carboxylic vibration also suggests a more symmetric bonding of the carboxylate group to lead. ${ }^{39,42,43}$ Oleate ligands are preferably bound to lead on a (111) facet. ${ }^{20}$ However, the density of lead atoms on a PbSe (111) facet is $6.16 \mathrm{~nm}^{-2}$ and exceeds the maximum packing fraction of hydrocarbon chains $(\approx 4-4.6$ $\left.\mathrm{nm}^{-2}\right) .{ }^{44,45}$ Most likely, some oleate must coordinate to two lead atoms to lower the steric hindering, thus forming a bridging coordination. The exchange of some oleate with iodide makes room to accommodate more oleate ligands to one single lead atom, which can explain the difference in the carboxylate coordinations that we see here.

Aliphatic Chain Structure. The $\mathrm{CH}_{2}$ bending vibrations $(\delta)$ in Figure 5a specify the crystallinity of the hydrocarbon chain. ${ }^{41,46,47}$ The progression of bands between 1150 and 1400 $\mathrm{cm}^{-1}$ is a series of well-defined peaks for all-trans crystalline chains and can be assigned to the twisting-rocking and wagging $\mathrm{CH}_{2}$ vibration. ${ }^{48,49}$ The $\mathrm{CH}_{2}$ groups can move freely in a liquidlike state, which results in a large number of rotational isomers resulting in the disappearance of the peaks for liquid chains. ${ }^{41,48,49}$ The spectrum of NCs with a hybrid ligand shell shows almost no sharp peaks, while the pristine NCs possess sharp and pronounced peaks. This shows that the aliphatic chains of the oleate ligands are more liquidlike (viz. more internal motion) in the hybrid ligand shell, while partly crystalline in the pristine PbSe NCs. In addition, also the peak position of the symmetric and asymmetric $\mathrm{CH}_{2}$ stretch in Figure $5 \mathrm{~b}$ can specify if the chain is crystalline (2850 and 2920 $\left.\mathrm{cm}^{-1}\right)$ or liquidlike $\left(2856\right.$ and $\left.2928 \mathrm{~cm}^{-1}\right) .{ }^{50,51}$ We therefore determined the accurate positions of the peaks by fitting them with Gaussians. Figure 6 shows the peak positions for untreated NCs in blue and for the chemical-treated NCs with a hybrid ligand shell in red. Despite the considerable spread of the data, the peak positions found for the hybrid ligand shell are at a higher wavenumber, thus indicating a more "liquidlike" structure compared with the hydrocarbon ligand layers on the pristine PbSe NCs. Besides the hybrid ligand shell, also the NC size and the ligand density influence the ligand crystallinity. Ligands have more space and are therefore more liquidlike if the ligand density is low or with increased surface curvature (smaller NCs). This is also visible here, where the low-density (LD, 2.2 oleate $\mathrm{nm}^{-2}$ ) and high-liganddensity (HD, 3.6 oleate $\mathrm{nm}^{-2}$ ) NCs with the same size are 


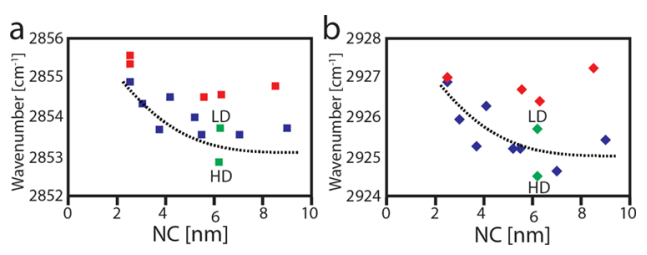

Figure 6. Structure of the oleate ligand shell for PbSe NCs with a hybrid iodide/oleate ligand shell (red data points) or original $\mathrm{PbSe}$ NCs with oleate ligands only (green and blue data points) measured from the $\mathrm{C}-\mathrm{H}$ stretch vibrations. (a) Position of the symmetric $\mathrm{C}-\mathrm{H}$ stretch vibration, where $2850 \mathrm{~cm}^{-1}$ is indicative of crystalline hydrocarbons and $2856 \mathrm{~cm}^{-1}$ of liquid hydrocarbons. For oleatecapped $\mathrm{PbSe} \mathrm{NCs}$, the vibration is at around $2852.5-2854 \mathrm{~cm}^{-1}$, indicative of an ordered hydrocarbon layer, while for the hybrid iodide-oleate ligand-capped $\mathrm{PbSe} \mathrm{NCs}$ (red), there is an upward shift to $2854-2856 \mathrm{~cm}^{-1}$, indicative of a more fluidlike capping layer. (b) Asymmetric stretch vibrations: stretch vibrations at $2920 \mathrm{~cm}^{-1}$ indicate a crystalline hydrocarbon layer, while those at $2926 \mathrm{~cm}^{-1}$ indicate a liquid hydrocarbon chain. The oleate-capped PbSe NCs show this vibration at around $2924-2926 \mathrm{~cm}^{-1}$. The PbSe NCs with a hybrid capping show the vibration at around $2927 \mathrm{~cm}^{-1}$, indicative of a more fluid capping layer. The green data points originate from the same $\mathrm{PbSe}$ NCs with a high (HD) or low (LD) ligand density.

illustrated in green. The liquid-to-solid phase change of ligands is important for the solubility ${ }^{52-54}$ and the NC self-assembly. The attractive interactions between ligands with a ligand shell and between adjacent NCs in an assembly sensitively influence the energy and structure of the self-assembly, especially in the absence of a good solvent. ${ }^{55}$

Recently, it was proposed that the broadening of the peaks in ${ }^{1} \mathrm{H}$ NMR gives an indication of solvent inclusion into the ligand shell. ${ }^{56}$ The features in NMR are indeed broader for the hybrid ligand shell (Figure S12). The broad signal also tells us that the $\mathrm{Pb}$ (oleate) $)_{2}$ ligands are not detached from the $\mathrm{NC}$ surface. $^{22}$ This is also confirmed by the absence of a very pronounced bridging coordination of the carboxylates, which is typical for free $\mathrm{Pb}$ (oleate) ${ }_{2}^{20}$ (see Figure S5). Based on the arguments presented above, we propose a sketch of the NC surface chemistry of the hybrid ligand shell compared to the normal ligand shell in Figure 7.

\section{CONCLUSIONS}

PbSe NCs can be made air-stable for several days using a postsynthetic treatment with $\mathrm{NaI}$ dissolved in methanol with
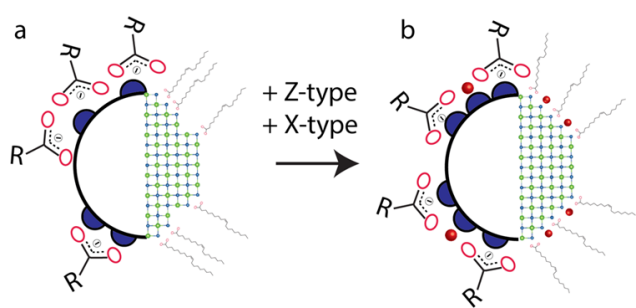

Figure 7. Representation of the change in surface chemistry for $\mathrm{PbSe}$ NCs capped with $\mathrm{Pb}$ (oleate) ${ }_{2}$ ligands (a) to $\mathrm{PbSe} \mathrm{NCs}$ capped with $\mathrm{Pb}$ (oleate) $)_{2}$ and $\mathrm{PbI}_{2}$ ligands (b). (a) Surface for pristine PbSe NCs. The oleate ligands are coordinated in bridging, and (a) symmetric chelating bidentate coordination and some hydrocarbon chains are packed together to illustrate the crystalline state. (b) Iodide/oleate hybrid ligand shell, with the iodide ions shown in red. The oleates are bound in (a) symmetric chelating coordinations and are more evenly distributed on the NC surface.
$\mathrm{Pb}$ (oleate) $)_{2}$ added to the $\mathrm{NC}$ dispersion. With the air-stable NCs as building blocks, superstructures can be obtained, however with a limited control of the geometry. It is advised to perform the chemical passivation with $\mathrm{NaI} / \mathrm{Pb}$ (oleate) ${ }_{2}$ after the formation of the superstructures. The oleate coordination on the $\mathrm{PbSe} \mathrm{NC}$ surface changes from a mixture of symmetric and asymmetric chelating bidentate and bridging carboxylate coordination to exclusively chelating bidentate coordinated oleates for chemically passivated samples. We also introduced an FT-IR spectroscopic method to measure the degree of molecular stiffness in the ligand layer. We show that after chemical passivation, in the mixed iodide/oleate ligand shell, the oleate ligands have more freedom of internal motion. Lead chalcogenide nanocrystals show strong potential for optoelectrical devices, such as development of cheap but sensitive photodetectors in the IR region. Our results show that air stability can be achieved without dramatic changes of the surface chemistry. This enables a much easier and cheaper processing of chalcogenide nanocrystals in the optoelectronic industry.

\section{CHEMICALS}

Lead acetate trihydrate ( $\geq 99.99 \%)$, 1-octadecene (90\%), oleic acid (90\%), selenium powder (100-mesh) (99.99\%), diphenylphosphine $(98 \%)$, lead fluoride $(\geq 99)$, lead chloride(II) $(99,999 \%)$, lead bromide $(99,999 \%)$, ammonium fluoride $(\geq 99.99)$, ammonium chloride $(\geq 99.5)$, ammonium bromide ( $\geq 99.99)$, ammonium iodide $(99,999 \%)$, cadmium fluoride $(99,8 \%)$, cadmium chloride $(99,99 \%)$, cadmium bromide $(99,999 \%)$, cadmium iodide $(99,999 \%)$, trioctylphosphine (90\%), tetrachloroethylene (TCE) ( $\geq 99 \%)$ (anhydrous), toluene (99.8\%) (anhydrous), methanol (99.8\%) (anhydrous), butanol (99.8\%) (anhydrous), cyclohexyl isocyanide (98\%), trifluoroacetic acid (99\%), trifluoroacetic anhydride $(\geq 99 \%)$, isopropanol (99.5\%) (anhydrous), triethylamine ( $\geq 99 \%)$, and pyrrolidine $(\geq 99 \%)$ were purchased from Sigma-Aldrich and used without further purification. Lead(II) oxide $(99.999+\%)$ was purchased from Strem. Hexadecane ( $\geq 99 \%)$, ethylene glycol (anhydrous, 99.8\%), and diphenyl ether ( $\geq 99 \%)$ were purchased from Sigma-Aldrich and degassed under vacuum for $\sim 14 \mathrm{~h}$ at $60{ }^{\circ} \mathrm{C}$ prior to use.

\section{EXPERIMENTAL SECTION}

The PbSe NCs were synthesized using two different procedures.

The first method was developed by Steckel et al. ${ }^{57}$ In brief, $4.77 \mathrm{~g}$ of $\mathrm{Pb}\left(\mathrm{C}_{2} \mathrm{H}_{3} \mathrm{O}_{2}\right)_{2} \cdot 3 \mathrm{H}_{2} \mathrm{O}, 10.35 \mathrm{~g}$ of oleic acid $(\mathrm{Pb} /$ oleic acid ratio of 1:2.91), and $39.75 \mathrm{~g}$ of 1-octadecene were mixed and degassed at 120 ${ }^{\circ} \mathrm{C}$ under vacuum for $5 \mathrm{~h}$ to remove water and acetate. The selenium precursor was made by dissolving $3.52 \mathrm{~g}$ of Se power in $0.41 \mathrm{~g}$ of diphenylphosphine and $46.59 \mathrm{~g}$ of trioctylphosphine. The selenium precursor was injected into a heated solution of the lead precursor $\left(180{ }^{\circ} \mathrm{C}\right)$ under vigorous stirring. After $70 \mathrm{~s}$, the reaction was quenched via injection of $30 \mathrm{~mL}$ of a methanol/butanol mixture (1:2). The mixture was centrifuged, and the black residue was dissolved in toluene $(10 \mathrm{~mL})$. Methanol was added $(8 \mathrm{~mL})$ to precipitate the suspension, which was subsequently centrifuged. The black residue was dissolved in toluene, and the cycle was repeated two more times.

The second method was developed by Campos et al. ${ }^{58}$ The lead oleate precursor was made via the method of Hendricks et al. ${ }^{59}$ The selenium precursor ( $N$-cyclohexylpyrrolidine-1-carboselenoamide) was prepared by mixing selenium $(9.0 \mathrm{mmol})$, pyrrolidine $(9.0$ $\mathrm{mmol})$, cyclohexyl isocyanide $(9.0 \mathrm{mmol})$, and $10 \mathrm{~mL}$ toluene. This mixture was heated to $\sim 100{ }^{\circ} \mathrm{C}$ until a clear solution was obtained. Extra pyrrolidine and/or cyclohexyl isocyanide was added if not all 
selenium reacted. The mixture was cooled down to room temperature, at which the selenourea precipitated. The liquid was decanted, and the obtained white solid was placed under vacuum for $24 \mathrm{~h}$. Subsequently, the solid was redissolved in $10 \mathrm{~mL}$ of toluene at $100{ }^{\circ} \mathrm{C}$ and cooled down. The precipitate was filtered, and the solid was placed under vacuum for another $24 \mathrm{~h}$.

The synthesis of the NCs was performed in a Schlenk line where $1.6 \mathrm{~g}$ (2.08 mmol, 1.2 equiv) of $\mathrm{Pb}$ (oleate) $)_{2}$ was dissolved in $140 \mathrm{~mL}$ of hexadecane in a $250 \mathrm{~mL}$ three-neck flask. Then, $0.45 \mathrm{~g}(1.7 \mathrm{mmol}$, 1 equiv) of $\mathrm{N}$-cyclohexylpyrrolidine-1-carboselenoamide was dissolved in $8 \mathrm{~mL}$ of diphenyl ether. Both precursors were heated to 100 ${ }^{\circ} \mathrm{C}$ to yield a clear colorless solution. The selenourea was quickly injected into the $\mathrm{Pb}$ (oleate) $)_{2}$ solution, which turned brown in approximately $8 \mathrm{~s}$. After $110 \mathrm{~min}$, the reaction was cooled down with an ice bath and introduced inside the glovebox. Furthermore, $90 \mathrm{~mL}$ of $n$-butanol and $60 \mathrm{~mL}$ of methanol were added to precipitate the NCs. The mixture was centrifuged, and the black residue was dissolved in $10 \mathrm{~mL}$ of toluene. This solution was washed three more times with methanol.

The $\mathrm{PbSe}$ NCs were made air-stable using $\mathrm{Pb}$ (oleate) ${ }_{2}$ dissolved in the NC solution and $\mathrm{NaI}$ dissolved in methanol. The chemical formula and concentration of the PbSe NCs were estimated from the NC size and FT-IR and NMR spectroscopy techniques, as shown in the main text. Extra $\mathrm{Pb}$ (oleate) ${ }_{2}$ (2.5 times the amount already present on the NC surface) was added and dissolved in the NC suspension in toluene $(10 \mathrm{~mL})$. For example, $0.5 \mathrm{~mL}$ of a $1.6 \mathrm{~nm}$ PbSe NC stock solution $\left(4.7 \times 10^{-5} \mathrm{M}\right)$ has, on average, 155 $\mathrm{Pb}$ (oleate $)_{2}$ ligands. $\mathrm{Pb}$ (oleate $)_{2}\left(9.1 \times 10^{-6} \mathrm{~mol}\right)$ was dissolved in 9.5 $\mathrm{mL}$ toluene, which was subsequently added to the NCs solution. This solution was stirred vigorously, and $\mathrm{NaI}\left(5.8 \times 10^{-6} \mathrm{~mol}\right)$ was added dropwise in the methanol solution $(0.5 \mathrm{~mL})$. Extra methanol was added after $2 \mathrm{~min}$ to precipitate the NCs. The mixture was centrifuged, and the black residue was collected and redispersed in toluene.

Several batches of PbSe NCs were used throughout the study, but for all samples, the change in the absorption peak position was checked.

FT-IR measurements were performed on a Bruker Vertex 70 spectrometer. A special air-tight liquid cell was used, purchased from International Crystal Laboratories, with a path length of $0.5 \mathrm{~mm}$ and two $\mathrm{KBr}$ crystals at the back and front side to make it transparent for IR light. Spectra where recorded from 400 to $7500 \mathrm{~cm}^{-1}$, with a $\mathrm{KBr}$ beam splitter, a DLaTGS D301 detector, and a mid-IR source. For all measurements, tetrachloroethylene (TCE) was used as solvent.

Ligand density was determined using FT-IR spectroscopy by a procedure published before. ${ }^{20}$ In short, a calibration curve of the integral of the $\mathrm{CH}$ stretch vibration was made from known oleic acid concentrations. PbSe NCs were dissolved in tetrachloroethylene, and an infrared absorption spectrum of the $400-7500 \mathrm{~cm}^{-1}$ region was recorded with an air-tight liquid cell. The exciton peak was integrated to determine the $\mathrm{NC}$ concentration and its peak position to determine the NCs size. ${ }^{60}$ By combining the NC size, the NC concentration, and the concentration of oleate, the amount of oleate per NC and the oleates per $\mathrm{nm}^{2}$ were calculated. Ligand density measurements were always combined with NMR to make sure that all oleate ligands were bound to the NC surface. ${ }^{21,22}$

The square superstructures were synthetized using a method adapted from the literature. ${ }^{9}$ A Petri dish was completely filled with $6.5 \mathrm{~mL}$ of EG, creating a flat surface. Subsequently, $350 \mu \mathrm{L}$ of a diluted NC solution $\left(7.7 \times 10^{-8} \mathrm{M}\right)$ in toluene was drop-casted on top of the $6.5 \mathrm{~mL} \mathrm{EG}$. The square structure was formed on top of the EG within approximately $30 \mathrm{~min}$ and could be transferred to any substrate. Small quantities of high-boiling-point organics (oleic acid, hexadecane) can increase the long-range order of the lattice. The whole experiment is performed in a glovebox containing $<0.1 \mathrm{ppm} \mathrm{O}_{2}$ and free from amines or other volatile gasses. The synthesis was successful for NCs with sizes in between 5 and $9 \mathrm{~nm}$. The oxygen level reported in Figure 3 was determined with an analog MBRAUN oxygen analyzer (\#2974).
${ }^{1} \mathrm{H}$ NMR measurements were performed using Agilent MRF400 equipped with a OneNMR probe and an Optima Tune system. Spectra were recorded according to the following parameters: 400 $\mathrm{MHz}, \mathrm{CDCl}_{3}, 25{ }^{\circ} \mathrm{C}$. Measurements of $\mathrm{PbSe} \mathrm{NCs}$ were performed using a longer relaxation delay $(30 \mathrm{~s})$ to allow complete relaxation. For $\mathrm{Pb}(\text { oleate })_{2}, \Delta=5.3(\mathrm{~m}, 4 \mathrm{H}, \mathrm{HC}=\mathrm{CH}), 2.2(\mathrm{t}, 4 \mathrm{H}, 3 \mathrm{JHH}=7.3$ $\left.\mathrm{Hz}, \mathrm{CH}_{2}\right), 2.0\left(\mathrm{~m}, 8 \mathrm{H}, \mathrm{CH}_{2}\right), 1.6\left(\mathrm{p}, 4 \mathrm{H}, 3 \mathrm{JHH}=7.8 \mathrm{~Hz}, \mathrm{CH}_{2}\right), 1.4-$ $1.1\left(\mathrm{~m}, 42 \mathrm{H}, \mathrm{CH}_{2}\right), 0.87\left(\mathrm{t}, 6 \mathrm{H}, 3 \mathrm{JHH}=6.7 \mathrm{~Hz}, \mathrm{CH}_{3}\right)$

TEM images where obtained using a Philips Tecnai microscope operating at $200 \mathrm{kV}$.

EDX spectra were obtained by a Philips Tecnai microscope operating at $200 \mathrm{kV}$.

XPS samples for XPS measurements were prepared by drop-casting NC solutions in toluene on aluminum plates, in a glovebox. Sample shipping, handling, and loading to the XPS apparatus were done under an inert gas atmosphere. XP spectra were recorded on a Thermo Scientific K-Alpha spectrometer equipped with a monochromatic small-spot X-ray source and a $180^{\circ}$ double-focusing hemispherical analyzer with a 128-channel delay line detector. Spectra were obtained using an aluminum anode $(\mathrm{Al} \mathrm{K} \alpha=1486.6 \mathrm{eV}$ ) operated at $72 \mathrm{~W}$ and a spot size of $400 \mu \mathrm{m}$. Survey scans were measured at a constant pass energy of $200 \mathrm{eV}$, and high-resolution scans of the separate regions were measured at $50 \mathrm{eV}$ pass energy. The background pressure of the ultrahigh vacuum chamber was $<2 \times 10^{-8}$ mbar. Sample charging was compensated for by the use of an electron flood gun, and binding energy (BE) calibration was done by setting the $\mathrm{C} 1 \mathrm{~s}$ peak of $\mathrm{sp}^{3}$ carbon to $\mathrm{BE}(\mathrm{C} 1 \mathrm{~s})=284.8 \mathrm{eV}$.

\section{ASSOCIATED CONTENT}

\section{S Supporting Information}

The Supporting Information is available free of charge on the ACS Publications website at DOI: 10.1021/acs.chemmater.9b01891.

Air-stable PbSe NCs; TEM images of original and airstable PbSe NCs, superstructure stabilized by $\mathrm{NH}_{4} \mathrm{I}$ and $\mathrm{CdI}_{2}$; EDX spectrum; XPS survey and HR-XPS iodide spectra; FT-IR spectrum of $\mathrm{Pb}$ (oleate) $)_{2}$, hydroxide contamination; square superstructure synthesized from air-stable NCs; absorption peak shifts; asymmetric and symmetric carboxylic stretch in FT-IR spectra; peak positions; and NMR spectra (PDF)

\section{AUTHOR INFORMATION}

\section{Corresponding Author}

*E-mail: d.vanmaekelbergh@uu.nl.

ORCID $\odot$

D. Vanmaekelbergh: 0000-0002-3535-8366

\section{Author Contributions}

The manuscript was written through contributions of all authors. All authors have given approval to the final version of the manuscript.

\section{Notes}

The authors declare no competing financial interest.

\section{ACKNOWLEDGMENTS}

D.V. acknowledges support from NWO physics (FOM program 152) and from the ERC grant, grant 692691-FIRST STEP.

\section{ABBREVIATIONS}

NC, nanocrystal; EDX, energy-dispersive X-ray spectrum; XPS, $\mathrm{X}$-ray photoelectron spectroscopy; FT-IR, Fourier transform infrared; NMR, nuclear magnetic resonance 


\section{REFERENCES}

(1) Kang, I.; Wise, F. W. Electronic Structure and Optical Properties of PbS and PbSe Quantum Dots. J. Opt. Soc. Am. B 1997, 14, 16321646.

(2) Kulkarni, A.; Evers, W. H.; Tomić, S.; Beard, M. C.; Vanmaekelbergh, D.; Siebbeles, L. D. A. Efficient Steplike Carrier Multiplication in Percolative Networks of Epitaxially Connected PbSe Nanocrystals. ACS Nano 2017, 12, 378-384.

(3) Midgett, A. G.; Luther, J. M.; Stewart, J. T.; Smith, D. K.; Padilha, L. A.; Klimov, V. I.; Nozik, A. J.; Beard, M. C. Size and Composition Dependent Multiple Exciton Generation Efficiency in $\mathrm{PbS}, \mathrm{PbSe}$, and $\mathrm{PbSxSe} 1-\mathrm{x}$ Alloyed Quantum Dots. Nano Lett. 2013, 13, 3078-3085.

(4) Davis, N. J. L. K.; Böhm, M. L.; Tabachnyk, M.; WisniveskyRocca-Rivarola, F.; Jellicoe, T. C.; Ducati, C.; Ehrler, B.; Greenham, N. C. Multiple-Exciton Generation in Lead Selenide Nanorod Solar Cells with External Quantum Efficiencies Exceeding 120\%. Nat. Commun. 2015, 6, No. 8259.

(5) Ning, Z.; Voznyy, O.; Pan, J.; Hoogland, S.; Adinolfi, V.; Xu, J.; Li, M.; Kirmani, A. R.; Sun, J.-P.; Minor, J.; Kemp, K. W.; Dong, H.; Rollny, L.; Labelle, A.; Carey, G.; Sutherland, B.; Hill, I.; Amassian, A.; Liu, H.; Tang, J.; Bakr, O. M.; Sargent, E. H. Air-Stable n-Type Colloidal Quantum Dot Solids. Nat. Mater. 2014, 13, 822-828.

(6) Ip, A. H.; Thon, S. M.; Hoogland, S.; Voznyy, O.; Zhitomirsky, D.; Debnath, R.; Levina, L.; Rollny, L. R.; Carey, G. H.; Fischer, A.; Kemp, K. W.; Kramer, I. J.; Ning, Z.; Labelle, A. J.; Chou, K. W.; Amassian, A.; Sargent, E. H. Hybrid Passivated Colloidal Quantum Dot Solids. Nat. Nanotechnol. 2012, 7, 577-582.

(7) Boneschanscher, M. P.; Evers, W. H.; Geuchies, J. J.; Altantzis, T.; Goris, B.; Rabouw, F. T.; van Rossum, S. A. P.; van der Zant, H. S. J.; Siebbeles, L. D. A.; Van Tendeloo, G.; Swart, I.; Hilhorst, J.; Petukhov, A. V.; Bals, S.; Vanmaekelbergh, D. Long-Range Orientation and Atomic Attachment of Nanocrystals in 2D Honeycomb Superlattices. Science 2014, 344, 1377-1380.

(8) Peters, J. L.; Altantzis, T.; Lobato, I.; Jazi, M. A.; van Overbeek, C.; Bals, S.; Vanmaekelbergh, D.; Sinai, S. B. Mono- and Multilayer Silicene-Type Honeycomb Lattices by Oriented Attachment of PbSe Nanocrystals: Synthesis, Structural Characterization, and Analysis of the Disorder. Chem. Mater. 2018, 30, 4831-4837.

(9) van Overbeek, C.; Peters, J. L.; van Rossum, S. A. P.; Smits, M.; van Huis, M. A.; Vanmaekelbergh, D. Interfacial Self-Assembly and Oriented Attachment in the Family of $\mathrm{PbX}(\mathrm{X}=\mathrm{S}, \mathrm{Se}, \mathrm{Te})$ Nanocrystals. J. Phys. Chem. C 2018, 122, 12464-12473.

(10) Tadjine, A.; Delerue, C. Colloidal Nanocrystals as LEGO Bricks for Building Electronic Band Structure Models. Phys. Chem. Chem. Phys. 2018, 20, 8177-8184.

(11) Kalesaki, E.; Delerue, C.; Morais Smith, C.; Beugeling, W.; Allan, G.; Vanmaekelbergh, D. Dirac Cones, Topological Edge States, and Nontrivial Flat Bands in Two-Dimensional Semiconductors with a Honeycomb Nanogeometry. Phys. Rev. X 2014, 4, No. 011010.

(12) Sykora, M.; Koposov, A. Y.; McGuire, J. A.; Schulze, R. K.; Tretiak, O.; Pietryga, J. M.; Klimov, V. I. Effect of Air Exposure on Surface Properties, Electronic Structure, and Carrier Relaxation in PbSe Nanocrystals. ACS Nano 2010, 4, 2021-2034.

(13) Zhang, Y.; Dai, Q.; Li, X.; Cui, Q.; Gu, Z.; Zou, B.; Wang, Y.; $\mathrm{Yu}, \mathrm{W}$. W. Formation of PbSe/CdSe Core/Shell Nanocrystals for Stable near-Infrared High Photoluminescence Emission. Nanoscale Res. Lett. 2010, 5, 1279-1283.

(14) Zhang, J.; Gao, J.; Church, C. P.; Miller, E. M.; Luther, J. M.; Klimov, V. I.; Beard, M. C. PbSe Quantum Dot Solar Cells with More than 6\% Efficiency Fabricated in Ambient Atmosphere. Nano Lett. 2014, 14, 6010-6015.

(15) Bae, W. K.; Joo, J.; Padilha, L. A.; Won, J.; Lee, D. C.; Lin, Q.; Koh, W.; Luo, H.; Klimov, V. I.; Pietryga, M. Highly Effective Surface Passivation of PbSe Quantum Dots through Reaction with Molecular Chlorine. J. Am. Chem. Soc. 2012, 134, 20160-20168.

(16) Zhang, J.; Gao, J.; Miller, E. M.; Luther, J. M.; Beard, M. C. Diffusion-Controlled Synthesis of $\mathrm{PbS}$ and $\mathrm{PbSe}$ Quantum Dots with in Situ Halide Passivation for Quantum Dot Solar Cells. ACS Nano 2014, 8, 614-622.

(17) Zhang, Z.; Liu, C.; Zhao, X. Utilizing Sn Precursor To Promote the Nucleation of $\mathrm{PbSe}$ Quantum Dots with in Situ Halide Passivation. J. Phys. Chem. C 2015, 119, 5626-5632.

(18) Woo, J. Y.; Ko, J. H.; Song, J. H.; Kim, K.; Choi, H.; Kim, Y. H.; Lee, D. C.; Jeong, S. Ultra-Stable PbSe Nanocrystal Quantum Dots via in Situ Formation of Atomically Thin Halide Adlayers on PbSe(100). J. Am. Chem. Soc. 2014, 136, 8883-8886.

(19) Brown, P. R.; Kim, D.; Lunt, R. R.; Zhao, N.; Bawendi, M. G.; Grossman, J. C.; Bulovic, V. Energy Level Modification in Lead Sulfide Quantum Dot Thin Films through Ligand Exchange. ACS Nano 2014, 8, 5863-5872.

(20) Peters, J. L.; van den Bos, K. H. W.; Van Aert, S.; Goris, B.; Bals, S.; Vanmaekelbergh, D. Ligand-Induced Shape Transformation of PbSe Nanocrystals. Chem. Mater. 2017, 29, 4122-4128.

(21) Anderson, N. C.; Hendricks, M. P.; Choi, J. J.; Owen, J. S. Ligand Exchange and the Stoichiometry of Metal Chalcogenide Nanocrystals: Spectroscopic Observation of Facile Metal-Carboxylate Displacement and Binding. J. Am. Chem. Soc. 2013, 135, 1853618548.

(22) Moreels, I.; Fritzinger, B.; Martins, J. C.; Hens, Z. Surface Chemistry of Colloidal PbSe Nanocrystals. J. Am. Chem. Soc. 2008, 130, 15081-15086.

(23) Liu, H.; Guyot-Sionnest, P. Photoluminescence Lifetime of Lead Selenide Colloidal Quantum Dots. J. Phys. Chem. C 2010, 114, 14860-14863.

(24) Weser, U.; Sokolowski, G.; Piltz, W. Reaction of Selenite with Biochemically Active Thiols: An X-Ray Photoelectron Spectroscopic Study. J. Electron Spectrosc. Relat. Phenom. 1977, 10, 429-439.

(25) Malmsten, G.; Thorén, I.; Högberg, S.; Bergmark, J.-E.; Karlsson, S.-E.; Reban, E. Selenium Compounds Studied by Means of ESCA. Phys. Scr. 1971, 3, 96-100.

(26) Shenasa, M.; Sainkar, S.; Lichtman, D. XPS Study of Some Selected Selenium Compounds. J. Electron Spectrosc. Relat. Phenom. 1986, 40, 329-337.

(27) Tomaev, V. V.; Makarov, L. L.; Tikhonov, P. A.; Solomennikov, A. A. Oxidation Kinetics of Lead Selenide. Glass Phys. Chem. 2004, 30, 349-355.

(28) Pederson, L. R. Two-Dimensional Chemical-State Plot for Lead Using XPS. J. Electron Spectrosc. Relat. Phenom. 1982, 28, 203-209.

(29) Hwang, G. W.; Kim, D.; Cordero, J. M.; Wilson, M. W. B.; Chuang, C.-H. M.; Grossman, J. C.; Bawendi, M. G. Identifying and Eliminating Emissive Sub-Bandgap States in Thin Films of $\mathrm{PbS}$ Nanocrystals. Adv. Mater. 2015, 27, 4481-4486.

(30) Taylor, J. A.; Perry, D. L. An X-ray Photoelectron and Electron Energy Loss Study of the Oxidation of Lead. J. Vac. Sci. Technol., A 1984, 2, No. 771.

(31) Baker, J. M.; Johnson, R. W.; Pollak, R. A. Surface Analysis of Rf Plasma Oxidized In and PbInAu Films Using ESCA. J. Vac. Sci. Technol. 1979, 16, No. 1534.

(32) Zherebetskyy, D.; Scheele, M.; Zhang, Y.; Bronstein, N.; Thompson, C.; Britt, D.; Salmeron, M.; Alivisatos, A. P.; Wang, L.-W. Hydroxylation of the Surface of $\mathrm{PbS}$ Nanocrystals Passivated with Oleic Acid. Science 2014, 344, 1380-1384.

(33) Cao, Y.; Stavrinadis, A.; Lasanta, T.; So, D.; Konstantatos, G. The Role of Surface Passivation for Efficient and Photostable PbS Quantum Dot Solar Cells. Nat. Energy 2016, 1, No. 16035.

(34) Jo, J. W.; Choi, J.; Garc, F. P.; Sei, A.; Sun, B.; Kim, Y.; Ahn, H.; Fan, J.; Quintero-bermudez, R.; Kim, J.; Choi, M.; Baek, S.; Proppe, A. H.; Walters, G.; Nam, D.; Kelley, S.; Hoogland, S.; Voznyy, O.; Sargent, E. H. Acid-Assisted Ligand Exchange Enhances Coupling in Colloidal Quantum Dot Solids. Nano Lett. 2018, 18, 4417-4423.

(35) Geuchies, J. J.; van Overbeek, C.; Evers, W. H.; Goris, B.; de Backer, A.; Gantapara, A. P.; Rabouw, F. T.; Hilhorst, J.; Peters, J. L.; Konovalov, O.; Petukhov, A. V.; Dijkstra, M.; Siebbeles, L. D. A.; van Aert, S.; Bals, S.; Vanmaekelbergh, D. In Situ Study of the Formation Mechanism of Two-Dimensional Superlattices from PbSe Nanocrystals. Nat. Mater. 2016, 15, 1248-1254. 
(36) Ghosh, S.; Manna, L. The Many “ Facets” of Halide Ions in the Chemistry of Colloidal Inorganic Nanocrystals. Chem. Rev. 2018, 118, 7804-7864.

(37) Cass, L. C.; Malicki, M.; Weiss, EA. The Chemical Environments of Oleate Species within Samples of Oleate-Coated PbS Quantum Dots. Anal. Chem. 2013, 85, 6974-6979.

(38) Deacon, G. B.; Philiips, R. J. Relationships between the CarbonOxygen Stretching Frequencies of Carboxylato Complexes and the Type of Carboxylate Coordination. Coord. Chem. Rev. 1980, 33, 227250.

(39) Tackett, J. E. FT-IR Characterization of Metal Acetates in Aqueous Solution. Appl. Spectrosc. 1989, 43, 483-489.

(40) Wang, Y.; Du, X.; Guo, L.; Liu, H. Chain Orientation and Headgroup Structure in Langmuir Monolayers of Stearic Acid and Metal Stearate (Ag, Co, $\mathrm{Zn}$, and $\mathrm{Pb}$ ) Studied by Infrared ReflectionAbsorption Spectroscopy. J. Chem. Phys. 2006, 124, No. 134706.

(41) Peters, J. L.; de Wit, J.; Vanmaekelbergh, D. Sizing Curve, Absorption Coefficient, Surface Chemistry and Aliphatic Chain Structure of PbTe Nanocrystals. Chem. Mater. 2019, 31, 1672-1680.

(42) Ellis, H. A.; White, N. A. S.; Taylor, R. A.; Maragh, P. T. Infrared, X-Ray and Microscopic Studies on the Room Temperature Structure of Anhydrous Lead (II) n-Alkanoates. J. Mol. Struct. 2005, 738, 205-210.

(43) Darensbourg, D. J.; Holtcamp, M. W.; Longridge, E. M.; Klausmeyer, K. K.; Reibenspies, J. H. Structural Characterization of Bidentate Carboxvlate Derivatives of Copper (I) Bistriphenylphosphine*. Inorg. Chim. Acta 1994, 227, 223-232.

(44) Strong, L.; Whitesides, G. M. Structures of Self-Assembled Monolayer Films of Organosulfur Compounds Adsorbed on Gold Single Crystals: Electron Diffraction Studies. Langmuir 1988, 4, 546558.

(45) Ulman, A. Formation and Structure of Self-Assembled Monolayers. Chem. Rev. 1996, 96, 1533-1554.

(46) Hostetler, M. J.; Stokes, J. J.; Murray, R. W. Infrared Spectroscopy of Three-Dimensional Self-Assembled Monolayers: NAlkanethiolate Monolayers on Gold Cluster Compounds. Langmuir 1996, 12, 3604-3612.

(47) Catalano, J.; Murphy, A.; Yao, Y.; Yap, G. P. A.; Zumbulyadis, N.; Centeno, A.; Dybowski, C. Coordination Geometry of Lead Carboxylates - Spectroscopic and Crystallographic Evidence. Dalton Trans. 2015, 44, 2340-2347.

(48) Maroncelli, M.; Qi, S. P.; Strauss, H. L.; Snyder, R. G. Nonplanar Conformers and the Phase Behavior of Solid N-Alkanes. J. Am. Chem. Soc. 1982, 104, 6237-6247.

(49) Snyder, R. G. Vibrational Study of the Chain Conformation of the Liquid N-Paraffins and Molten Polyethylene. J. Chem. Phys. 1967, 47, 1316-1360.

(50) Snyder, R. G.; Strauss, H. L.; Elliger, C. A. C-H Stretchlng Modes and the Structure of n-Alkyl Chains. 1. Long, Disordered Chains. J. Phys. Chem. A 1982, 86, 5145-5150.

(51) Templeton, A. C.; Hostetler, M. J.; Kraft, C. T.; Murray, R. W. Reactivity of Monolayer-Protected Gold Cluster Molecules: Steric Effects. J. Am. Chem. Soc. 1998, 120, 1906-1911.

(52) Yang, Y.; Qin, H.; Jiang, M.; Lin, L.; Fu, T.; Dai, X.; Zhang, Z.; Niu, Y.; Cao, H.; Jin, Y.; Zhao, F.; Peng, X. Entropic Ligands for Nanocrystals: From Unexpected Solution Properties to Outstanding Processability. Nano Lett. 2016, 16, 2133-2138.

(53) Yang, Y.; Qin, H.; Peng, X. Intramolecular Entropy and SizeDependent Solution Properties of Nanocrystal - Ligands Complexes. Nano Lett. 2016, 16, 2127-2132.

(54) Geyer, T.; Born, P.; Kraus, T. Switching Between Crystallization and Amorphous Agglomeration of Alkyl Thiol-Coated Gold Nanoparticles. Phys. Rev. Lett. 2012, 109, No. 128302.

(55) Ang, T. P.; Wee, T. S. A.; Chin, W. S. Three-Dimensional SelfAssembled Monolayer (3D SAM) of n-Alkanethiols on Copper Nanoclusters. J. Phys. Chem. B 2004, 108, 11001-11010.

(56) De Roo, J.; Yazdani, N.; Drijvers, E.; Lauria, A.; Maes, J.; Owen, J. S.; Van Driessche, I.; Niederberger, M.; Wood, V.; Martins, J. C.; Infante, I.; Hens, Z. Probing Solvent-Ligand Interactions in Colloidal
Nanocrystals by the NMR Line Broadening. Chem. Mater. 2018, 30, 5485-5492.

(57) Steckel, J. S.; Coe-Sullivan, S.; Bulović, V.; Bawendi, M. G. $1.3 \mu \mathrm{m}$ to $1.55 \mu \mathrm{m}$ Tunable Electroluminescence from $\mathrm{PbSe}$ Quantum Dots Embedded within an Organic Device. Adv. Mater. 2003, 15, $1862-1866$

(58) Campos, M. P.; Hendricks, M. P.; Beecher, A. N.; Walravens, W.; Swain, R. A.; Cleveland, G. T.; Hens, Z.; Sfeir, M. Y.; Owen, J. S. A Library of Selenourea Precursors to PbSe Nanocrystals with Size Distributions near the Homogeneous Limit. J. Am. Chem. Soc. 2017, 139, 2296-2305.

(59) Hendricks, M. P.; Campos, M. P.; Cleveland, G. T.; Jen-La Plante, I.; Owen, J. S. A Tunable Library of Substituted Thiourea Precursors to Metal Sulfide Nanocrystals. Science 2015, 348, 12261230.

(60) Moreels, I.; Lambert, K.; De Muynck, D.; Vanhaecke, F.; Poelman, D.; Martins, J. C.; Allan, G.; Hens, Z. Composition and Size-Dependent Extinction Coefficient of Colloidal PbSe Quantum Dots. Chem. Mater. 2007, 19, 6101-6106. 